\title{
Qualidade de vida de residentes em condomínio exclusivo para idosos
}

\author{
Juliana Lima ${ }^{1}$ Elen Ferraz Teston² Sonia Silva Marcon ${ }^{3}$
}

\section{RESUMO}

Objetivo: comparar a qualidade de vida de idosos que residem sozinhos com a de idosos que moram com companheiro. Método: Estudo quantitativo, realizado junto à totalidade de idosos (50) residentes no Condomínio do Idoso de Maringá-PR, os quais foram divididos em dois grupos, sendo o grupo 1 constituído por idosos que moram só (28) e o grupo dois por 11 casais, totalizando 22 idosos. Os dados foram coletados entre novembro de 2011 e fevereiro de 2012, com aplicação dos instrumentos WHOQOL-OLD e o WHOQOL-BREF. Resultados: A percepção de qualidade de vida é relativamente alta nos dois grupos. Os escores relacionados ao domínio psicológico e faceta intimidade foram significativamente maior entre os idosos que residem com companheiro (p: 0,$001 ; p: 0,002$, respectivamente). Conclusão: Os profissionais de saúde devem identificar as necessidades dos idosos que moram só relacionadas a estas variáveis com vistas à melhora da qualidade de vida.

Descritores: Idoso; Qualidade de vida; Família.

\section{Quality of life among older people who live alone and accompanied: comparative study}

\begin{abstract}
Objective: To compare the quality of life of elderly people who live alone with the elderly who live with a partner. Method: Quantitative study, conducted by the total number of elderly (50) Condo residents in the Elderly Maringá-PR, which were divided into two groups, group 1 consists of elderly people who live alone (28) and two per group 11 couples, totaling 22 seniors. Data were collected between November 2011 and February 2012, with implementation of the WHOQOL-OLD and WHOQOL-BREF. Results: The perception of quality of life is relatively high in both groups. The scores related to psychological intimacy and facet area were significantly higher among the elderly living with a partner (p: 0.001, p: 0.002, respectively). Conclusion: Health professionals should identify the needs of older people living alone related to these variables in order to improve the quality of life.
\end{abstract}

Descriptors: Aged; Quality of Life; Family.

\footnotetext{
${ }^{1}$ Especialização em andamento em Gestão em Saúde na Universidade Estadual de Maringá (UEM), Maringá, PR, Brasil.

${ }^{2}$ Doutoranda em Enfermagem na Universidade Federal de Maringá (UEM), Maringá, PR, Brasil.

${ }^{3}$ Doutora em Filosofia da Enfermagem pela Universidade Federal de Santa Catarina (UFSC), Florianópolis, SC, Brasil.
} 


\section{Introdução}

Há cerca de quatro décadas constata-se o aumento mundial da população idosa. O Brasil é um exemplo típico dessa afirmativa, pois o crescimento do envelhecimento populacional é exponencial e a projeção para o ano de 2025 mostra que o número de indivíduos com idade igual ou superior a 60 anos será de 32 milhões. As regiões Sul e Sudeste do País tem a maior proporção de indivíduos com 60 anos ou mais, com índices de 12,7\% e 12,3\%, respectivamente ${ }^{1}$.

Diante desse aumento, as redes de suporte e apoio a esta faixa etária precisam ser estruturadas, seja no âmbito social, econômico ou político. Uma forma de organização são os atuais condomínios para idosos, nova modalidade habitacional para idosos de baixa renda, que se constituem em uma estratégia de garantia do direito à moradia àqueles que vivem em condições precárias. Esses condomínios, além de possibilitarem o direito de uma habitação digna, também prezam pela manutenção da qualidade de vida (QV) de seus habitantes².

Qualidade de vida refere-se à "percepção do indivíduo sobre sua posição na vida, no contexto da cultura e sistemas de valores nos quais vive, e em relação aos seus objetivos, expectativas, padrões e preocupações". Trata-se de um conceito amplo e complexo que engloba a saúde física, o estado psicológico, o nível de independência, as relações sociais, as crenças pessoais e a relação com as características do meio ambiente ${ }^{3}$.

Outro fenômeno observado é o crescimento do número de domicílios unipessoais formados por idosos. Deste modo, conhecer o universo dos idosos que moram sozinhos torna-se uma questão importante, visto que a família exerce papel considerável no bem-estar e QV dos idosos ${ }^{4}$.

Salienta-se que nessa etapa da vida os indivíduos geralmente ficam mais susceptíveis à necessidades de suporte funcional, financeiro e também emocional ${ }^{5}$. Destarte, idosos que vivem com outras pessoas, parentes ou não, podem ser melhor amparados em situações adversas. Em contrapartida, idosos que moram sozinhos são considerados mais vulneráveis e desprovidos de apoio diante das dificuldades. Ressalta-se ainda que, com o aumento na expectativa de vida, cresce a probabilidade de os indivíduos idosos necessitarem de assistência e apoio financeiro da família para continuar desenvolvendo suas atividades, o que demanda tempo e alocação de recursos ${ }^{4}$. Diante disso, surgem alguns questionamentos sobre a influencia da co-residência sobre a QV dos indivíduos idosos.

Ao se constatar, na literatura científica, a escassez de pesquisas que retratem a QV de pessoas que residem nesse tipo de moradia específica para o idoso e, considerando ainda a existência de diferenças nos tipos de arranjos domiciliares pergunta-se: Idosos residentes nessa nova modalidade de habitação que residem com companheiro apresentam diferença na percepção da QV em relação àqueles que residem sozinhos? Em quais aspectos? Sob essa ótica, a presente investigação pretendeu ampliar o conhecimento sobre essa temática e favorecer a proposição de ações aos residentes nesses condomínios, a fim de promover melhorias na atenção que lhes é dispensada no âmbito da saúde coletiva e na QV.Diante do exposto definiu-se como objetivo deste estudo comparar a QV de idosos que residem sozinhos com a de idosos que moram com companheiro.

\section{Metodologia}

Estudo transversal de abordagem quantitativa, desenvolvido junto a idosos residentes no Condomínio do Idoso do município de Maringá-PR.

Participaram do estudo os 50 idosos residentes no condomínio, sendo eles divididos em grupo 1 (G1) composto por 28 idosos que moram só e grupo 2 (G2) por 11 casais,totalizando 22 idosos neste grupo.

O único critério para a inclusão no estudo foi obter pontuação mínima de 13 pontos no Miniexame do Estado Mental (MEEM), instrumento de medida do funcionamento cognitivo geral de rastreio e não diagnóstico.

Os dados foram coletados nos domicílios dos idosos no período de novembro de 2011 a fevereiro de 2012, por meio de entrevista semiestruturada individual abordando características sociodemográficas e aplicação dos instrumentos WHOQOL-BREF e o WHOQOL-OLD.

O WHOQOL-BREF é um instrumento de avaliação da QV proposta pela Organização Mundial de Saúde (OMS), já validado no Brasil, constituído de 26 questões, sendo duas referentes a QV geral e as demais contemplam os domínios físico, psicológico, relações sociais e meio ambiente. As respostas a cada questão são apresentadas em uma escala do tipo likert de cinco pontos com escores totais variando entre 38 a 118 pontos. Vale lembrar que na avaliação dos 
resultados as questões 03,04 e 26 devem ser interpretadas de forma inversa. Esse processo é necessário para não haver problemas na interpretação dos escores, pois o instrumento avalia QV e suas variáveis de maneira positiva e crescente $^{6}$.

O WHOQOL-OLD por sua vez é constituído de 24 pontos e as facetas avaliados por ele são: funcionamento dos sentidos, autonomia, atividades passadas, presentes e futuras; participação social; morte e morrer e intimidade. As respostas a cada questão também são apresentadas em uma escala do tipo likert variando de 1 a 5, sendo sete questões com interpretação negativa: 1, 2, 6, 7, 8, 9, e 107. Os escores totais variam entre 52 e 102.

Ambos os instrumentos não preconizam ponto de corte para classificar a QV, como ótima, boa, ou ruim, sendo definido apenas que quanto mais alto o escore de QV melhor a percepção desta.

Os instrumentos de QV são autoaplicáveis, porém, em razão da possível dificuldade de leitura, problemas visuais e analfabetismo entre os idosos, optou-se por realizar entrevista direta com todos os participantes, os quais foram orientados a responder as perguntas do questionário tomando como base as duas semanas anteriores à data da realização da coleta dos dados, conforme orientação de uso dos dois instrumentos.

Para análise dos resultados, os dados foram digitados em planilha eletrônica do programa EXCEL e submetidos à análise descritiva por meio da freqüência simples. Cada domínio do WHOQOL-BREF e cada faceta do WHOQOL-OLD foram isoladamente consolidados no Software Statistical Package for the Social Sciences (SPSS) com as respectivas sintaxes e analisados. Como os dados não seguem distribuição normal utilizou-se a mediana para representar os dados, e estatística não-paramétrica para comparar a QV entre os dois grupos por meio do teste Mann Whitney. Para todas as análises foi considerado o intervalo de confiança de $95 \%$ e o nível de significância de 5\%. Dessa forma, apresentaramse os percentis 2,5\% e 97,5\% para apresentar os limites inferior e superior da distribuição dos dados considerando a confiança de $95 \%$.

O desenvolvimento do estudo ocorreu em conformidade com o preconizado pela resolução $n^{0} 466 / 2012$ do Conselho Nacional de Saúde que disciplina pesquisa com seres humanos. O projeto foi aprovado pelo Comitê Permanente de Ética em Pesquisa com Seres Humanos da Universidade Estadual de Maringá, (parecer $n^{0}$ 70912011). Todos os participantes foram devidamente orientados sobre o propósito do estudo e atestaram sua anuência em participar do mesmo assinando o Termo de Consentimento Livre e Esclarecido (TCLE) em duas vias.

\section{Resultados}

Dos 50 idosos em estudo, a maioria era do sexo feminino (62\%), tinha idade entre 60 a 79 anos (86\%), renda mínima de um salário mínimo (90\%) e baixa escolaridade, sendo $30 \%$ deles analfabetos e $36 \%$ com no máximo quatro anos de estudo. A totalidade dos idosos referiu seguir uma religião, em especial a católica (68\%).

A percepção de QV a partir dos dois instrumentos utilizados é relativamente alta nos dois grupos, visto que nenhum dos domínios ou faceta teve mediana inferior a 60 pontos.

Em relação à avaliação da QV mensurada pelo WHOQOL-BREF, o domínio que apresentou maior escore para os idosos do G1 foi o psicológico sendo o limite inferior e superior da distribuição dos dados referentes a este domínio 58.33 e 87.50, respectivamente. Já para os idosos do G2 o maior escore foi o das relações sociais, sendo 41.67 e 91.67 os limites inferior e superior, respectivamente. Cabe salientar que os escores para o domínio físico foi igual nos dois grupos e para os outros três domínios eles foram maiores para os idosos com companheiro. Esta diferença, no entanto foi significativa somente em relação ao domínio psicológico.

A QV avaliada pelo WHOQOL-OLD evidenciou maior escore, para os dois grupos, nas facetas: funcionamento dos sentidos, participação social e morte e morrer. Verificou-se ainda, que o escore de QV referente ao domínio psicológico (Med: 72.91; p: 0,001) e faceta intimidade (Med: 68.75; p:0,002) é significativamente maior entre os idosos do G2 em relação aos idosos do G1, conforme Tabela 1. 
Tabela 1 - Distribuição dos escores de QV dos residentes no Condomínio do idoso WHOQOL-BREF e WHOQOL-OLD, Maringá-Paraná, 2012.

\begin{tabular}{|c|c|c|c|c|c|c|c|}
\hline \multirow[t]{2}{*}{ Variáveis } & \multicolumn{3}{|c|}{$\begin{array}{l}\text { Idosos que residem sozinhos } \\
\text { (28) }\end{array}$} & \multicolumn{3}{|c|}{$\begin{array}{l}\text { Idosos que residem com companheiro } \\
\qquad(22)\end{array}$} & \multirow[t]{2}{*}{$p$} \\
\hline & Med $^{*}$ & Perc $^{* *} 2,5$ & Perc 97,5 & Med & Perc 2,5 & Perc 97,5 & \\
\hline \multicolumn{8}{|l|}{ WHOQOL-BREF } \\
\hline Físico & 60.71 & 51.70 & 80.36 & 60.71 & 32.14 & 85.71 & 0,288 \\
\hline Psicológico & 70.83 & 58.33 & 87.50 & 72.91 & 50.00 & 91.25 & 0,001 \\
\hline Relações Sociais & 66.67 & 58.33 & 91.67 & 75.00 & 41.67 & 91.67 & 0,133 \\
\hline Meio Ambiente & 65.83 & 60.94 & 75.69 & 68.75 & 50.00 & 87.34 & 0,553 \\
\hline \multicolumn{8}{|l|}{ WHOQOL-OLD } \\
\hline Funcionamento dos sentidos & 93.75 & 70.31 & 97.30 & 87.50 & 81.25 & 99.37 & 0,184 \\
\hline Autonomia & 62.50 & 56.25 & 93.44 & 62.50 & 56.25 & 92.50 & 0,327 \\
\hline Atividades passadas, presentes e futuras & 62.50 & 56.25 & 87.19 & 75.00 & 62.50 & 93.12 & 0,234 \\
\hline Participação social & 75.00 & 68.75 & 93.50 & 75.00 & 62.50 & 97.30 & 0,331 \\
\hline Morte e morrer & 75.00 & 68.75 & 87.19 & 75.00 & 68.75 & 93.12 & 0,553 \\
\hline Intimidade & 56.25 & 37.50 & 99.69 & 68.75 & 43.75 & 98.75 & 0,002 \\
\hline
\end{tabular}

*Mediana; ${ }^{* *}$ Percentil;

Teste Mann-Whitney

\section{Discussão}

Os resultados apontam que entre os idosos há uma superioridade no percentual de mulheres, sendo que há 3,9 milhões de mulheres a mais que homens no Brasil ${ }^{8}$, fato que pode ser justificado pelo aumento da expectativa de vida feminina nas últimas décadas.

No presente estudo observa-se o predomínio de idosos com até 79 anos $(86 \%)$, o que pode estar relacionado com alguns critérios de moradia no condomínio, como por exemplo, a independência funcional. Entretanto, o maior número de indivíduos idosos nessa faixa etária é uma característica do processo de envelhecimento brasileiro, diferente do que vem ocorrendo nos países desenvolvidos, onde a concentração é maior no grupo de 80 anos ou mais 9 .

Para os idosos de baixa renda, como é o caso no presente estudo, a existência do apoio social é fundamental para aumentar a capacidade de sobrevivência em situações adversas e melhoria na QV ${ }^{10}$.

A baixa escolaridade também foi evidenciada em estudo cujo objetivo foi comparar a $Q V$ de idosos de uma comunidade de Porto Alegre, RS, com a de idosos institucionalizados no interior de Minas Gerais ${ }^{11}$. Deve-se considerar ainda o fato de que estes idosos nasceram e cresceram em um período em que a dificuldade de acesso à educação era muito grande. Esses resultados ainda são reflexos dos baixos níveis educacionais vigentes no Brasil até recentemente ${ }^{12}$.

Aadesão da totalidade dos entrevistados à prática religiosa constitui fator importante para manutenção da QV, haja vista que a aproximação a práticas religiosas durante a velhice é fonte importante de suporte emocional, com repercussões nas áreas da saúde física e mental ${ }^{13}$. Acredita-se que a religiosidade na terceira idade age como instrumento facilitador no processo de adaptação as perdas, mudanças físicas e diante das doenças, desempenhando um papel relevante na recuperação e manutenção da saúde e QV ${ }^{14}$.

Observou-se que no domínio psicológico, o qual inclui fatores como auto-estima, capacidade de pensamento, imagem corporal, espiritualidade e crenças pessoais, um maior escore de QV esteve significativamente associado a idosos que moram com companheiro. Esse resultado está em concordância com os achados evidenciados em um outro estudo, no qual idosos que viviam com companheiro relataram melhor $\mathrm{QV}^{15}$. Isto pode ser justificado pelo fato de que o idoso que mora só está mais propenso ao desenvolvimento do sentimento de solidão, insatisfação pessoal e isolamento social, quando comparados com os idosos que moram com companheiro, além do que a presença da família constitui importante fonte de ajuda em todos os momentos da vida, o que é essencial ao bem estar ${ }^{16}$.

$\mathrm{Na}$ faceta intimidade, em que se avalia a capacidade de desenvolver relacionamentos pessoais e íntimos, verificouse maior escore de QV significativamente associado também a idosos que moram com companheiro. Considera-se que esta condição facilita a construção de laços afetivos aumentando a probabilidade de expressar sentimentos de companheirismo e amor pela vida. Este resultado corrobora o resultado de estudo realizado junto a idosos do município 
de Foz do Iguaçu, o qual apontou que os idosos que moram com até duas pessoas demonstraram maior percepção de QV referente a esta faceta ${ }^{16}$.

Apesar de não ter sido verificado diferença estatisticamente significativa entre as outras variáveis e o escore de QV, alguns aspectos são relevantes como os escores médios de QV elevados para ambos os grupos no domínio relações sociais e facetas, participação social, morte e morrer e funcionamento dos sentidos.

O convívio social é fundamental para que a pessoa viva melhor em qualquer fase da vida, o que tem sido associado ao aumento do senso de bem-estar e à melhora no funcionamento físico ${ }^{17}$. Portanto, há necessidade de criação de programas de incentivo à participação dos idosos em atividades comunitárias que lhes permitam cada vez mais o desenvolvimento de novas habilidades e a manutenção e o estímulo ao convívio social.

Os resultados satisfatórios da QV referente à relação e participação social, podem estar relacionados ao fato dos idosos viverem em um ambiente onde as relações com vizinhos são estimuladas à medida que várias atividades são realizadas em grupo semanalmente, promovidas pela Secretaria de assistência social do município, conforme relato dos entrevistados. Ademais, viver em um condomínio específico para idosos tem sido associado à maior possibilidade de interação social e bem-estar em termos de redução da solidão e ansiedade ${ }^{18}$. Isto permite inferir que os condomínios favorecem o estabelecimento de uma rede social de convivência — estratégia facilitadora da manutenção da QV.

A média dos escores encontrados em ambos os grupos, em relação às inquietações e temores sobre a morte e morrer foi satisfatória. Isto indica que os idosos não possuem uma visão negativa sobre o processo da morte ou estão, de certa forma, preparados para morrer, e isto reflete em sua QV. Este dado é semelhante ao encontrado em um estudo que associou esses resultados a questões de espiritualidade, pois os idosos, em geral creem que a morte é certa para todos, e que não se deve temê-la e sim aceitá-la ${ }^{19}$.

A variável funcionamento dos sentidos apresentou escore médio elevado para os idosos dos dois grupos, o que pode estar associado a um dos quesitos necessários para morar no condomínio - a independência para as atividades da vida diária. Em um estudo internacional ${ }^{20}$, foi investigado a percepção dos moradores sobre a missão dos condomínios, identificando-se que, para eles, um dos objetivos é a manutenção da sua autonomia.

Diante disso, destaca-se a importância de se identificar os fatores que possivelmente podem interferir no bem-estar e QV dos idosos, objetivando criar ações que busquem atender as necessidades dessa população, por meio da promoção da saúde e também da manutenção de sua QV.

Neste sentido, é importante poder contar com alguém que se disponha a escutar, dialogar e, muitas vezes, ajudar a compreender, solucionar ou mesmo aceitar os acontecimentos diários. Em se tratando de indivíduos que estão vivenciando a velhice, estágio de vida em que há redução da rede social advindo da saída dos filhos de casa, aposentadoria ou viuvez, a necessidade de socializar os fatos corriqueiros da vida incentivam a busca por relações de amizade reciprocamente satisfatórias. A interação social vivenciada pelos idosos colabora para o exercício de sua cidadania e, também, para que o indivíduo se sinta valorizado e inserido no meio social.

\section{Considerações finais}

Os resultados do estudo mostram que os idosos que moram com companheiro apresentam melhores escores de $\mathrm{QV}$ quando comparados com idosos que residem só, em relação ao domínio psicológico e faceta intimidade. Neste sentido, os profissionais de saúde devem identificar as necessidades dos idosos que moram só relacionadas a estas variáveis com vistas ao planejamento de ações que contribuam com a promoção e melhora da QV.

Evidenciou-se também, escores satisfatórios para os dois grupos, referentes às relações e participações sociais, funcionamento dos sentidos e morte e morrer, o que pode estar relacionado à oferta de atividades e promoção do convívio social que esta modalidade de habitação oferece.

Portanto, considerando que a avaliação do indivíduo com relação à QV pode variar de acordo com a constituição do domicílio, ambiente de moradia, tempo e com as prioridades ao longo da vida, sugere-se reflexão das equipes de saúdes com relação às ações desenvolvidas até o momento para a promoção da QV. 


\section{Referências bibliográficas}

1. Instituto Brasileiro de Geografia e Estatística (IBGE). Sobre a condição de saúde dos idosos: indicadores selecionados. Indicadores Sociodemográficos e de Saúde no Brasil. Brasília, DF, 2009. [acesso em: 17 set. 2012]. Disponível em: http:// www.ibge.gov.br/home/estatistica/populacao/indic_sociosaude/2009/com_sobre.pdf

2. Deus SAl. Um modelo de moradia para idosos: o caso da Vila dos Idosos do Pari São Paulo. Caderno Temático Kairós Gerontologia. 2010; 195-213.

3. Campo MO, Rodrigues Neto JFR. Qualidade de vida, um instrumento para promoção da saúde. Ver. Baiana Saúde Pública. 2008 ago; 32(2): 232-40.

4. Camargos MCS. Enfim só: um olhar sobre o universo de pessoas idosas que moram sozinhas no município de Belo Horizonte (MG) [tese]. Minas Gerais: Universidade Federal de Minas Gerais; 2008. 126 p.

5. Camargos MCS, Rodrigues RN, Machado CJ. Idoso, família e domicílio: uma revisão narrativa sobre a decisão de morar sozinho. Rev. Bras. Estud. Popul. 2011; 28(1): 217:30.

6. Fleck MPA, Louzada S, Xavier M, Chachamovich E, Vieira G, Santos L et al. Aplicação da versão em português do instrumento abreviado de avaliação da qualidade de vida "WHOQOL-bref. Rev Saúde Pública. 2000; 34(2): 706-16.

7. Fleck MPA, Chachamovich E, Trentini C. Development and validation of the Portuguese version of the WHOQOL-OLD module. Rev. Saúde Pública. 2006; 40(5):785-91.

8. Instituto Brasileiro de Geografia e Estatística (IBGE). Censo 2010: população do Brasil é de 190.732 .694 pessoas. [acesso em 13 de agosto de 2012] Disponível em: http://www.ibge.gov.br/home/presidencia/noticias/noticia_visualiza. php?id_noticia $=1766$

9. Sousa AI, Silver LD. Perfil sociodemográfico e estado de saúde autoreferido entre idosas de uma localidade de baixa renda. Esc Anna Nery. 2008; 12(4): 706-16.

10. Sousa Al, Silver LD, Griep RH. Apoio social entre idosas de uma localidade de baixa renda no município do Rio de Janeiro. Acta Paul. Enferm. 2010; 23(5): 625-31.

11. Vitorino LM, Paskulin LMG, Vianna LAC. Qualidade de vida de idosos da comunidade e de instituições de longa permanência: estudo comparativo. Rev. Latino Am. Enfermagem [Internet]. Jan/fev. 2013 [acesso em 13 de agosto de 2012]. Disponível em: http://www.scielo.br/pdf/rlae/v21nspe/pt_02.pdf

12. Diniz MA, Tavares DMS, Rodrigues LR. Características sociodemográficas e de saúde entre idosos com hipertensão arterial. Cienc. Cuid. Saúde. 2009. 8(4):607-14.

13. Floriano PJ, Dalgalarrondo P. Saúde mental, Qualidade de Vida e religião dos Idosos de um Programa de Saúde da Família. J. Bras Psiquiatr. 2007; 56(3). [acesso em 13 agosto de 2012]. Disponível em: http://www.scielo.br/scielo. php?script=sci_arttext\&pid=S0047-20852007000300002

14. Dias DSG, Carvalho CS, Araújo CV. Comparação da percepção subjetiva de qualidade de vida e bem-estar de idosos que vivem sozinhos, com a família e institucionalizados. Rev. Bras. Geriatr. Gerontol. 2013; 16(1): 127-38.

16. Falcade BL, Leite MT, Hildebrandt LM, et al. Octogenários em residências unipessoais: enfoque sobre a qualidade de vida e condições de saúde. Rev. Enferm. UFSM 2011;1(3):386-93.

17. Faller JW, Melo WA, Versa GLGS, Marcon SS. Qualidade de vida de idosos cadastrados na estratégia saúde da família de Foz do Iguaçu-PR. Esc. Anna Nery. 2010; 14(4): 803-10.

18. Inouye K, Pedrazzani E. Intruction, social economic status and evaluation of some dimensions of octogenarians' quality of life. Rev Latino Am. Enf. 2007; 15 (spe): 742-47.

19. Litwin H, Shiovitz-Ezra. Social network type and subjective well-being in a national Sample of older Americans. Gerontologist. 2011; 51(3): 379-88.

20. Torres GV, Reis LA, Fernandes MH. Qualidade de vida e fatores associados em idosos dependentes em uma cidade do interior do Nordeste. J Bras Psiquiatr. 2009; 58(1): 39-44.

21. ScharlachA, Graham C, Lehning A. The "Village" Model:A Consumer-DrivenApproach for Aging in Place. Gerontologist. 2011;52(esp):418-27. 


\section{Juliana Cristina de Lima}

Endereço para correspondência - Rua Dom Pedro II. Bairro Centro, CEP 87270-000, Maringá, PR, Brasil. E-mail: julianalima1500@hotmail.

Lattes: http://lattes.cnpq.br/9405299352013804

Elen Ferraz Teston - elen-1208@hotmail.com

Sonia Silva Marcon - soniasilva.marcon@gmail.com

Recebido em 19 de junho de 2013.

Publicado em 12 de setembro de 2014. 
